\title{
Vortex states in layered mesoscopic superconductors
}

\author{
Chao-Yu Liu, G. R. Berdiyorov, and M. V. Milošević \\ Departement Fysica, Universiteit Antwerpen, Groenenborgerlaan 171, B-2020 Antwerpen, Belgium
}

(Received 9 June 2010; revised manuscript received 19 January 2011; published 31 March 2011)

\begin{abstract}
Within the Ginzburg-Landau theory, we study the vortex structures in three-dimensional anisotropic mesoscopic superconductors in the presence of a uniform magnetic field. Anisotropy is included through varied $T_{c}$ in different layers of the sample and leads to distinct differences in the vortex states and their free energy. Several unconventional states are found, some comprising vortex clusters or exhibiting asymmetry. In a tilted magnetic field, we found second-order transitions between different vortex states, although vortex entry is generally a first-order transition in mesoscopic samples. In multilayered samples the kinked vortex strings are formed owing to the competing interactions of vortices with Meissner currents and the weak-link boundaries. The length and deformation of vortex fragments are determined solely by the inclination and strength of applied magnetic field, and this lock-in does not depend on the degree of anisotropy between the superconducting layers.
\end{abstract}

DOI: 10.1103/PhysRevB.83.104524

PACS number(s): 74.25.Ha, 74.25.Dw, 74.78.Na, 74.78.Fk

\section{INTRODUCTION}

Since the discovery of high-temperature superconductors (HTSs), ${ }^{1}$ much attention has been paid to their distinctly anisotropic structure - a stack of the two-dimensional (2D) superconducting layers separated by insulating layers and coupled with each other by Josephson tunneling. To date, the Josephson effect in superconductors found various applications, such as in microwave receivers, superconducting quantum interference device (SQUID) magnetometers, and logic elements, to name a few. Often, such applications are better realized in a small-sized weak-link structures, i.e., Josephson junctions with non-tunnel-type conductivity, owing to the low capacitance of such a junction. ${ }^{2}$ A weak link is commonly achieved by two superconducting layers separated by a normal metallic layer, or still a superconductor but with weaker critical parameters than those of the linked layers.

The layered structure of the superconducting sample certainly has important effects on the structure and behavior of magnetic vortices, which in turn can strongly influence the properties of the sample. The vortex matter in layered superconductors with extreme anisotropy can be theoretically described by the Lawrence-Doniach (LD) model, ${ }^{3}$ in which the continuous three-dimensional (3D) vortex line is replaced by composite vortex structures dependent on the tilt angle and strength of the applied magnetic field. In the simplest cases, a field parallel to the superconducting layers will induce a triangular, or in a suitable geometric confinement, rectangular lattice ${ }^{4}$ of Josephson vortices between the layers. The field perpendicular to the layers will result in a triangular lattice of pancake vortices in the superconducting planes. The more complex cases, i.e., the vortex states in layered anisotropic superconductors under tilted magnetic field, have also been extensively studied, ${ }^{5-12}$ with the central issue mainly focusing on the flux-line structure and the phase transition between different vortex states as a function of the field tilt.

In this paper, we introduce the weakly linked layered structure in $3 \mathrm{D}$ mesoscopic samples, with the objective to understand the penetration of (tilted) magnetic field in such samples, and the formation and rearrangement of vortex states with respect to the superconducting layers and the sample boundaries. As opposed to HTSs, where a tilted magnetic field will result in a set of crossing lattices of qualitatively different pancake and Josephson vortices, our system is anisotropic with periodically modulated $T_{c}$, where a tilted magnetic field creates a set of composite but deformed ("kinked") vortices. We restrict ourselves to the stationary picture, but here the reported complexity of vortex states will also have direct implications on the dynamic response of layered mesoscopic samples. ${ }^{13-15}$

In this paper, as opposed to the LD approximation, we take into account the full $3 \mathrm{D}$ of the sample structure. Besides considering a layered superconductor, we add one more degree of complexity - the sample size and geometry. This is the core objective in mesoscopic superconductivity, where enhancement of critical parameters is found owing to quantum confinement. ${ }^{16-19}$ A significant portion of earlier works was also devoted to the topologic confinement of flux structures, ${ }^{20-24}$ and we focus our work on the similar, though more complex and $3 \mathrm{D}$, phenomenon. We analyze the vortex structure and distribution in a cubic sample with one or more weak links under (tilted) magnetic field by a 3D Ginzburg-Landau (GL) simulation, a proven phenomenological approach for studies of vortex matter in superconductors. ${ }^{18-20,22-31}$ For example, successful numerical simulations using 3D GL formalism were performed to study the vortex states ${ }^{26-29}$ as well as the calorimetric properties ${ }^{30}$ of cylindrical, spherical, and conical mesoscopic samples.

The paper is organized as follows. In Sec. II, we show the derived GL equations with an anisotropy function and explain the numerical method and procedure we use in the calculations. In Secs. III and IV, we analyze the results obtained for samples with one central weak link and with multiple weak links, respectively. Starting with the deformation of vortices inside the weak link, we discuss further the issues concerning the distribution, interaction, and structure of vortices in the sample. In Sec. IV, we also present the $M(H)$ magnetization curves of our samples, which are of relevance to experiments. Our results are finally summarized in Sec. V. 


\section{THEORETICAL FORMALISM}

We consider a cubic mesoscopic sample with one or more weakened superconducting regions as illustrated in Fig. 1. We implement the GL theory by solving the equations obtained by minimizing the following energy functional:

$$
\begin{aligned}
G_{s H}-G_{n H}= & \int\left[\alpha|\Psi|^{2}+\frac{1}{2} \beta|\Psi|^{4}+\frac{1}{2 m^{*}}\right. \\
& \left.\times\left|\left(-i \hbar \vec{\nabla}-\frac{e^{*}}{c} \vec{A}\right) \Psi\right|^{2}-\frac{(\vec{h}-\vec{H})^{2}}{4 \pi}\right] d V,
\end{aligned}
$$

where $\vec{H}$ denotes the applied magnetic field, and $\vec{h}$ is the total local field, including the response of the superconductor. We include the anisotropy in the functional through the GL coefficient $\alpha$, as $\alpha=\frac{1}{\zeta(x, y, z)} \alpha_{0}$. Recalling the temperature dependence of the parameter $\alpha$ in GL theory, the function $\zeta>$ 1 in this case would correspond to $\left(1-T / T_{c}\right) /\left(1-T / T_{c w}\right)$ with the lower critical temperature $T_{c w}<T_{c}$ in the regions with weak superconductivity. In the presence of such anisotropy, by minimization of Eq. (1), we obtain the following equations for order parameter and vector potential,

$$
\begin{gathered}
(-i \vec{\nabla}-\vec{A})^{2} \Psi=\Psi\left(\frac{1}{\zeta}-|\Psi|^{2}\right), \\
\vec{\nabla} \times \vec{\nabla} \times \vec{A}=\vec{j}=\frac{1}{2 i}\left(\Psi^{*} \vec{\nabla} \Psi-\Psi \vec{\nabla} \Psi^{*}\right)-|\Psi|^{2} \vec{A},
\end{gathered}
$$

with boundary conditions

$$
\left.\left(-i \hbar \vec{\nabla}-\frac{e^{*}}{c} \vec{A}\right) \Psi\right|_{\perp, \text { boundary }}=0 .
$$

The above equations are given in dimensionless form, where distances are measured in units of the coherence length $\xi=\hbar / \sqrt{-2 m^{*} \alpha_{0}}$, the order parameter in $\psi_{0}=\sqrt{-\alpha_{0} / \beta}$, and the vector potential in $c \hbar / e^{*} \xi$. The magnetic field is measured in units of the second critical magnetic field $H_{c 2}=c \hbar / e^{*} \xi^{2}=$ $\kappa \sqrt{2} H_{c}$ and free energy in $F_{0}=H_{c}^{2} V / 8 \pi$, where $H_{c}$ is the

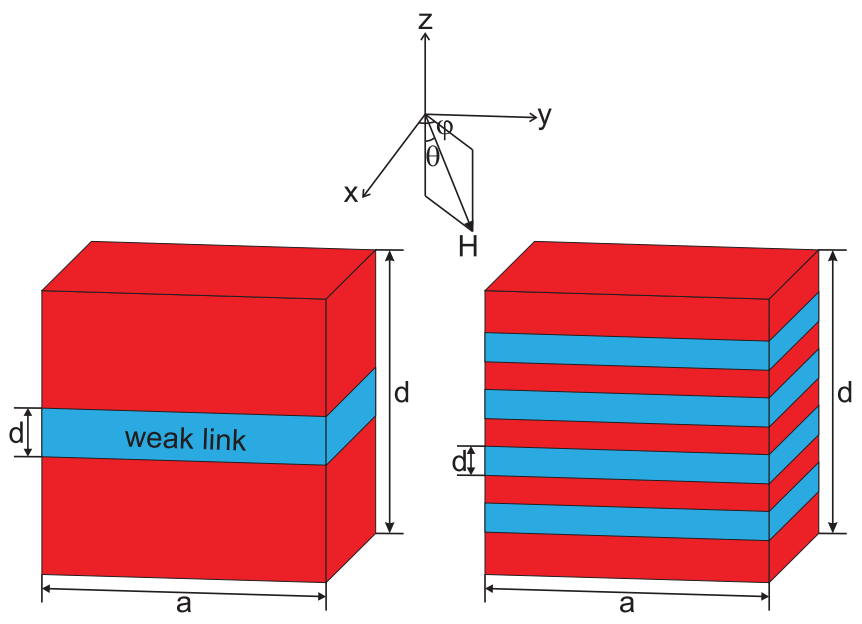

(a)

(b)

FIG. 1. (Color online) The considered system: a superconducting cube of lateral size $a$, with one (a) and four (b) weak links of thickness $d$, in the presence of a tilted magnetic field. thermodynamic critical field, $\kappa=\lambda / \xi$ is the GL parameter, and $\lambda=c \sqrt{m^{*} / \pi} / 2 e^{*} \psi_{0}$ is the magnetic-field penetration depth.

The weak link, i.e., the region in the sample with weak superconductivity, can be directly modeled by a decreased parameter $\alpha$, or in other words, the anisotropy function $\zeta$ larger than unity. For simplicity, we assume in this work a steplike behavior of $\zeta$ across the sample, so that it becomes a coefficient equal unity inside the superconducting layers, and larger than 1 inside the weak link(s). Then, for a fixed applied magnetic field, we solve the two coupled GL equations self-consistently using the Gauss-Seidel iterative procedure ${ }^{18}$ and the link variable approach $^{32}$ for a finite-difference representation of the order parameter and the vector potential on a uniform Cartesian space grid $(x, y, z)$ with a typical grid spacing of less than $\xi / 3$. Solving Eq. (3) with respect to the boundary condition, Eq. (4) enables us to calculate the full magnetic-field profile in and around the sample, and further determine the magnetization of the sample as

$$
M_{i}=\frac{H_{i}-\left\langle h_{i}\right\rangle}{4 \pi},
$$

with $H_{i}$ being the applied field and $\left\langle h_{i}\right\rangle$ denoting the averaged $i$-component of the local field in the measurement plane.

\section{MESOSCOPIC CUBE WITH A CENTRAL WEAK LINK}

In what follows, we first consider a superconducting cube of size $a=300 \mathrm{~nm}$ with a single weak link of size $d=30,50$, and $70 \mathrm{~nm}$, centered in the sample. As an example, we chose the coherence length $\xi=10 \mathrm{~nm}$ and penetration depth $\lambda \gg \xi$ so that the sample is an extreme type-II one. The anisotropy coefficient in the weak link was typically varied up to 10 in the simulations. It is important at this point to also define the direction of the applied field. Throughout the paper, the field direction will be specified by two angles $-\theta$, which is the angle with respect to the $z$ axis, and $\varphi$, which is the azimuthal angle in the $(x, y)$ plane with respect to the $x$ axis (as depicted in Fig. 1).

\section{A. A single weak link: Deformation of the vortex core}

To begin with, we discuss the deformation of the vortex core in the weak link, as illustrated in Fig. 2. The anisotropy, as included in our simulations, leads to the free-energy difference between the weak link in its superconducting and in its normal state smaller than that of the bulk superconductor (for $\zeta>1$ ). As a result, it is easier (less energetically costly) to suppress superconductivity inside a weak link than in the strongly superconducting layers. For that reason, the vortex core, as locally destroyed superconductivity, is more likely to reside inside the weak links than elsewhere in the sample. One should also note that the effective coherence length $\xi_{e}=\hbar / \sqrt{-2 m^{*} \alpha}$ is larger in the weak-link region owing to smaller $\alpha$. A larger coherence length in the weak link means that the superconducting order parameter recovers slower from 0 inside the vortex to its equilibrium value far from the vortex. In other words, the vortex core will expand inside the weak link (proportionally to $\zeta^{2}$ ), compared to the vortex size in the superconducting layers. However, the diffusion of 


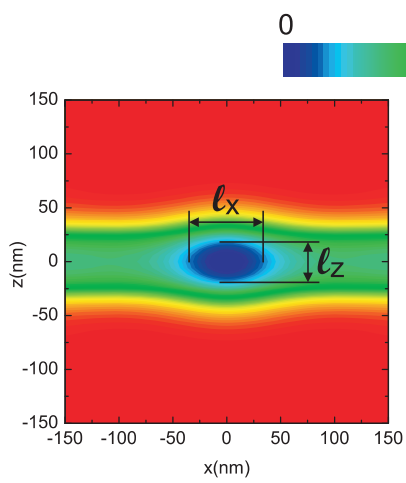

(a)

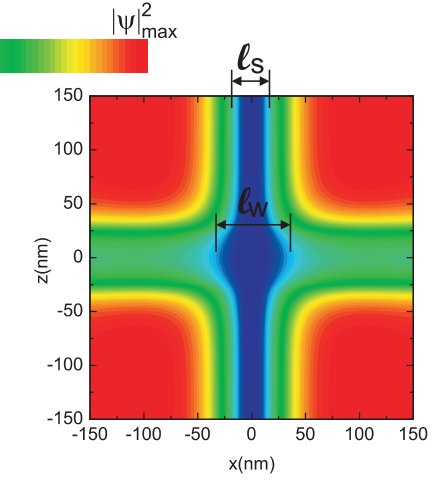

(b)

FIG. 2. (Color online) Contour plots of the Cooper pair density on the central $(x, z)$ plane of a sample with a weak link of thickness $50 \mathrm{~nm}$ and with an anisotropy coefficient $\zeta=10$, in a magnetic field applied (a) parallel and (b) perpendicular to the weak-link plane.

Cooper pairs from strongly superconducting regions into the weak link will also influence the deformation of the vortex core. Therefore, if the vortex currents are fully exploring the anisotropy of the sample, the $3 \mathrm{D}$ vortex tube can be strongly deformed.

To investigate this phenomenon in more detail, we first stabilized the $L=1$ (single vortex, $L$ being the vorticity) state in the sample. In an applied magnetic field parallel to the weak link, the vortex lies directly inside the weak link [see Fig. 2(a)]. Such a central location of the vortex is favorable in mesoscopic samples even in the absence of any weak links, but we note here the strong deformation of the vortex core, which is compressed between the superconducting layers (while actually expanding inside the weak link). Without the interaction with other vortices, we measured the ratio of the diameters of the vortex core in the directions parallel and perpendicular to the weak link as a function of anisotropy, as shown in Fig. 3(a). In this analysis, we define the size of the vortex core by a simple criterion of the rise of $|\Psi|^{2}$ to $10 \%$ of the equilibrium Cooper-pair density of the superconducting region in the particular direction. Figure 3(a) actually shows the $\zeta$ dependence of the deformation of the vortex core in the weak link for three different thicknesses of the weak link. In all cases, we conclude that the vortex core elongates along the weak link when anisotropy is increased. The deformation is expected to saturate at large anisotropy owing to the interaction of the vortex core with the lateral boundaries of the sample.

Similar vortex features were found in the case of a magnetic field applied perpendicularly to the weak-link plane [see Fig. 2(b)]. As explained earlier, a large anisotropy coefficient leads to a large effective coherence length in the weak link. As a consequence, the vortex tube expands inside the weak link owing to easily suppressed superconductivity. To illustrate this phenomenon, the ratios of the diameters of the vortex core inside the weak link (of different thicknesses) and on the top of the sample (fully superconducting region) were calculated as a function of anisotropy and shown in Fig. 3(b). As expected, the core inside the weak-link plane grows with increasing anisotropy. However, here the thickness of the weak link plays an important role, because the vortex tube is continuous between the strong and the weak superconducting region, and
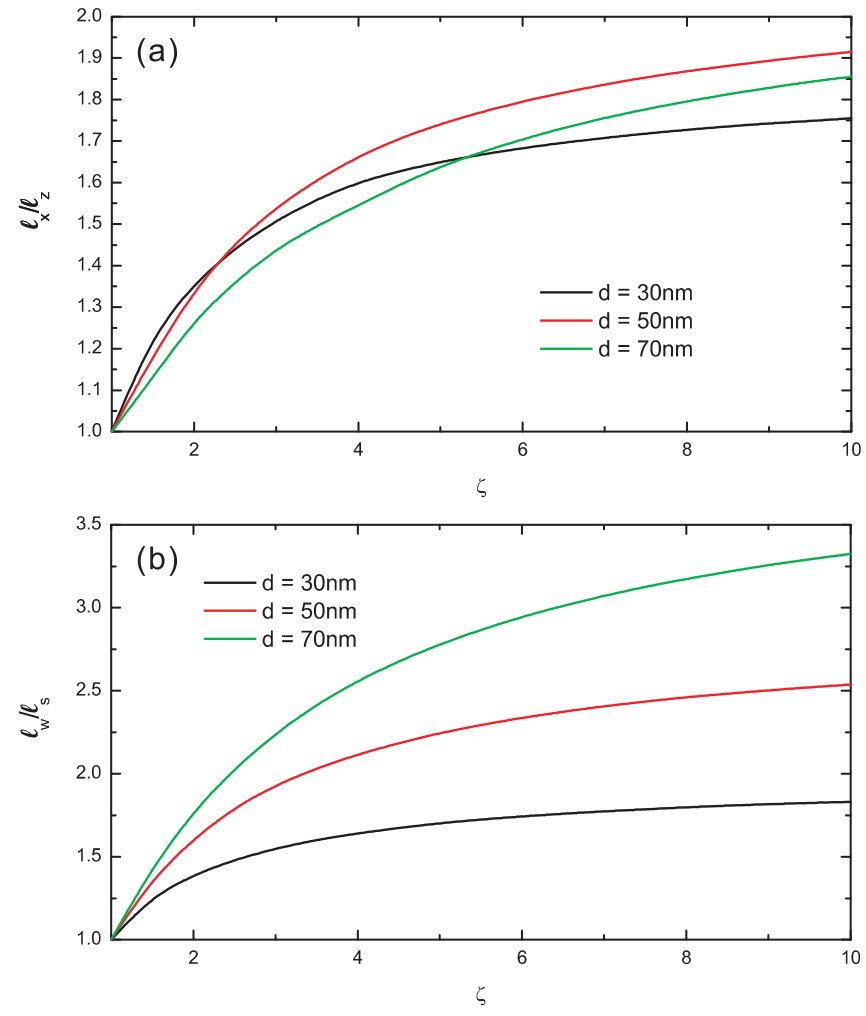

FIG. 3. (Color online) (a) Parallel field: The ratio of diameters of the (Josephson-like) vortex in directions across and along the weak link, as a function of the anisotropy, for three thicknesses of the weak link. (b) Perpendicular field: The ratio of diameters of the (Abrikosov) vortex core inside the weak link and on top of the sample.

the deformation of the core is also bound by the elasticity of the vortex tube. Therefore, for thin weak links, the vortex core is influenced more by the surrounding strong Meissner currents above and below the weak link than in the case of thicker weak links, and therefore the core deforms less.

\section{B. A single weak link: The vortex states}

After the analysis of the single-vortex state, we now calculate the full free-energy spectrum and the corresponding vortex states as a function of applied magnetic field for the sample with a central weak link of different thicknesses and anisotropy coefficients. In the first step, the magnetic field is kept parallel to the weak-link plane, and more specifically, parallel to the $y$ axis. For comparison, we also calculated the vortex states for a sample without any weak link, as shown in Fig. 4. The procedure for finding vortex states is multifold: (i) We first increase and decrease the magnetic field in the considered range, with a kept history of the previously found states in the field sweep; and (ii) for each value of the magnetic field, we also initialize the calculation from the fluctuating normal state (randomized $|\Psi|<0.01$ ) and from the fully superconducting state $(|\Psi| \approx 1)$. In such a way, we construct the energy diagram of all stable vortex states, even those with higher energy. As a result, we found that for the same vorticity of the vortex state, there can exist more than one stable vortex distribution, i.e., one with lowest energy (ground state) and several others with higher energy (usually called metastable). 

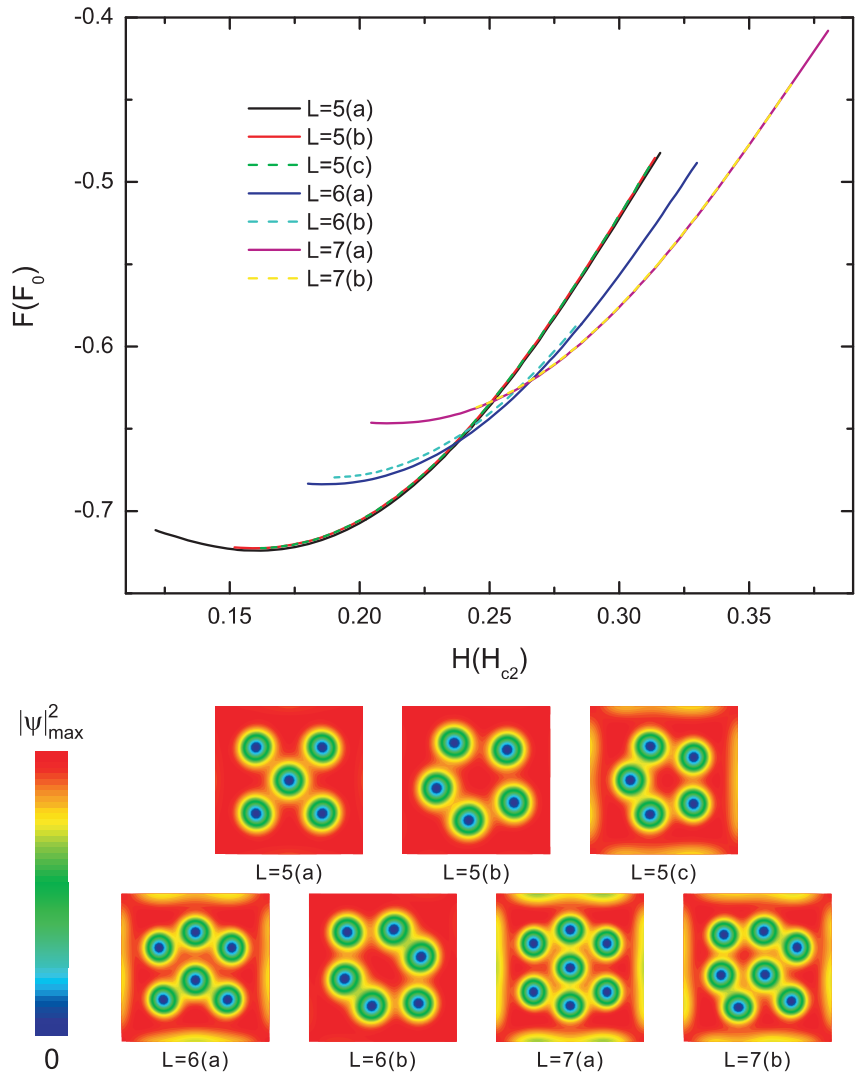

FIG. 4. (Color online) Free-energy curves and the corresponding vortex states in a sample without a weak link as a function of applied magnetic field parallel to the $y$ direction. Insets show the contour plots of the Cooper-pair density for selected different vortex states with the same vorticity.

In the insets of Fig. 4, we show three sets of such vortex states, for vorticities $L=5,6$, and 7 . The states obeying most the square symmetry of the sample are the lowest-energy ones, regardless of the vorticity, which is the conclusion that holds also in 2D mesoscopic polygons. ${ }^{22}$

This finding demonstrates the competition between the confinement imposed on vortices by Meissner currents and the vortex-vortex repulsion. The Meissner current is strongest at the edges of the sample and it confines vortices toward the interior of the sample. On the other hand, vortices are basically parallel magnetic moments that repel each other, and this repulsion, in the absence of sample boundaries, leads to the formation of a triangular (Abrikosov) lattice. For five vortices, the triangular lattice state is satisfied if vortices sit on apexes of a rectangle $a \times a \sqrt{3}$ with one vortex in the center. This is why the $L=5$ (a) square distribution in Fig. 4 has a lower energy and a higher stability than the also found pentagonal state, because it conforms both to the sample symmetry and closely to the low-energy triangular arrangement of vortices. For $L=6$, there are also two different states found. One is a diagonal-symmetric hexagonal configuration $[L=6(\mathrm{~b})]$, and the other is a zigzag structure $[L=6(\mathrm{a})]$. The latter is more reminiscent of a triangular lattice and therefore has lower energy. A similar conclusion supports the low energy of the twofold mirror-symmetric $L=7$ (a) state in Fig. 4, compared to the one with diagonal symmetry $[L=7(\mathrm{~b})]$.
Therefore, one concludes that the metastability of different vortex states results from a competition between the vortex interaction with Meissner currents (which actually represent the effect of sample boundaries) and the vortex-vortex interaction. However, when weak links are present in the sample, we add one more competing effect to this analysis, namely, the suppressed superconductivity in parts of the sample, where vortices favorably reside (minimize their energy). To begin with, we first consider a sample as in Fig. 4 but now with a central weak link of 50-nm thickness. The results are summoned in Fig. 5 for the anisotropy coefficients $\zeta=5$. As can be seen in these figures, vortices sit preferably inside the weak link until the saturation number is reached, i.e., there are enough vortices in the weak link so that the increased vortex-vortex interaction expels some of them into the fully superconducting part of the sample. Note also that vortices not only favorably reside in the weak link to minimize energy, but they also enter the sample through the weak link owing to the lower-energy barrier for vortex entry there.

In what follows, we will address different vortex distributions found for the same vorticity, shown as insets in Fig. 5. For an anisotropy coefficient $\zeta=5$, up to $L=3$, all vortices reside inside the weak link. However, three possible vortex states were found for the $L=4$ state. In one, all vortices lie within the weak link, and this state is stable in the range of fields from $0.164 H_{c 2}$ to $0.243 H_{c 2}$. The second $L=4$ state is asymmetric, and stable in the field range from $0.144 H_{c 2}$ to $0.229 H_{c 2}$. The third is another symmetric state with two vortices in the weak link, two vortices outside, and stable in the

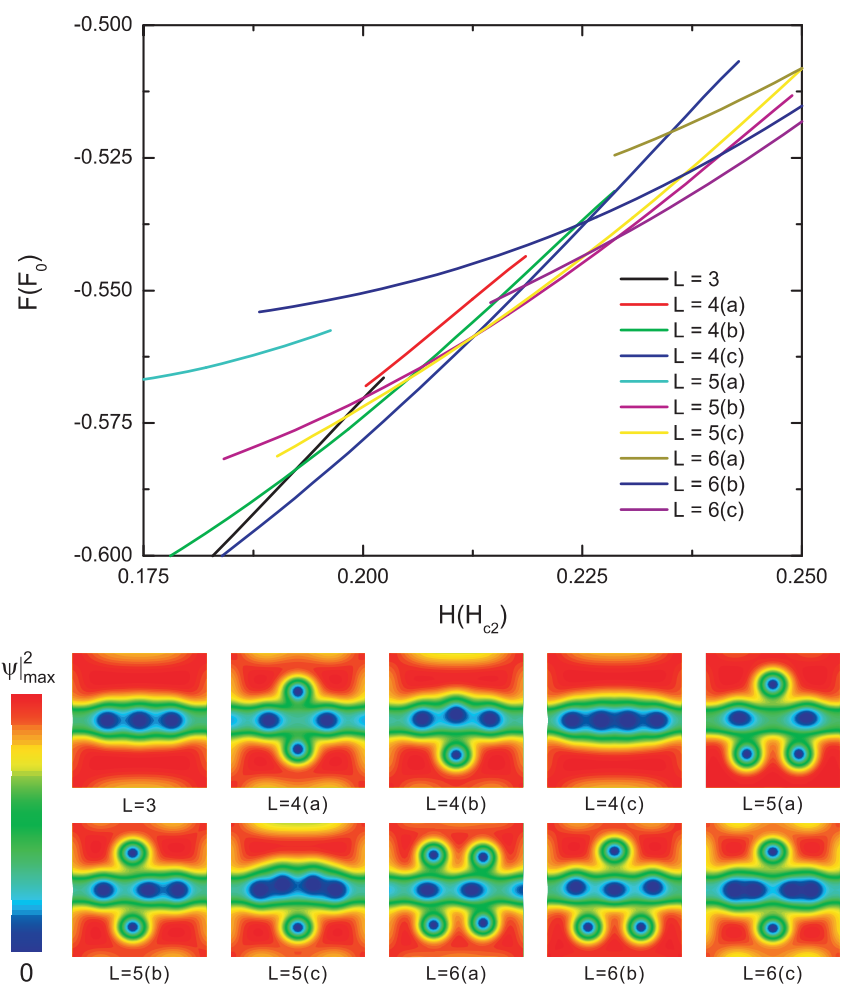

FIG. 5. (Color online) Free-energy curves and the corresponding vortex states in a sample with one central weak link of $50 \mathrm{~nm}$ and an anisotropy coefficient $\zeta=5$ in a magnetic field applied parallel to the $y$ direction. The insets show the contour plots of the Cooper-pair density in the central $(x, z)$ plane for selected vortex states. 
range of fields from $0.200 H_{c 2}$ to $0.219 H_{c 2}$. The asymmetric state, in which one vortex is expelled from the weak link, is the second lowest-energy one. Vortex-vortex interactions inside the weak link expel one vortex out to the strongly superconducting region. Although this allows, to some extent, for the triangulation of the vortex arrangement, the interaction between vortices and the Meissner current is increased in this state. Because the weak link is located in the center of the sample, the excess vortex must choose a preferential side to go away from it. As a consequence, the symmetry is broken, and an asymmetric vortex state is formed, which cannot be found in an isotropic superconducting cube. Note, however, that a similar phenomenon can be observed in 3D superconductors with a hole for a vorticity beyond the saturation vorticity of the hole. ${ }^{24}$ There, the first excess vortex after the saturation of the hole is reached must also break the symmetry, even though nothing in the theoretical formalism breaks the symmetry.

However, such an asymmetric vortex structure is less stable as a function of applied field than the linear one, not only because of the asymmetry, but also because of the increasing confinement. With increasing magnetic field, the asymmetric state loses stability in competition with encircling Meissner currents, which are growing stronger. Ultimately, all four vortices are compressed inside the weak link (as a first-order transition). When the anisotropy coefficient was increased to 10 , we found the state with even five vortices inside the weak link without any vortex inside the strongly superconducting regions, whereas a maximum of four vortices were found inside the weak link in Fig. 5. In other words, the increased anisotropy increases the energy gain for a vortex residing in the weak link, overpowering the loss in energy owing to unfavorable vortex-vortex repulsion.

The $L=4$ state is not the only vortex state exhibiting asymmetry in this system. For example, in Fig. 5 three possible configurations were found for both the $L=5$ and $L=6$ states. For $L=5$, one state $[L=5(\mathrm{c})]$ is similar to the state $L=4(\mathrm{~b})$, with one vortex residing outside and others residing inside the weak link. In the second allotropic configuration for $L=5$, three vortices fit inside the weak link, and two extra vortices are located, one at each side of the link. The vortices inside the fully superconducting regions occupy central positions owing to strong interactions with the Meissner currents. However, they also interact with vortices inside the weak link, particularly with the central vortex. To minimize this interaction, the central vortex shifts inside the weak link, breaking the symmetry now along the $x$ direction. This state decreases the repulsion among vortices without greatly increasing the interaction of vortices with Meissner currents and is therefore the lowest-energy state in a relatively high magnetic field. The third state with five vortices, $L=5(\mathrm{a})$, is a pentagonal structure that resembles the pentagonal vortex state in the solid cube (Fig. 4), but with two vortices inside the weak link.

A similar breaking of the vortex pattern inside the weak link is found for $L=6$ in the ground state, with four vortices inside the weak link and an additional one on each side. The enforced central position of the vortices in the fully superconducting regions causes the rearrangement of vortices inside the weak link [state $L=6$ (c) in Fig. 5]. Namely, two vortex dimers form inside the weak link, avoiding the central position (which is usually the most favorable position for a vortex in a symmetric mesoscopic superconductor). This shows that vortices may conglomerate inside the weak link to preserve the overall symmetry and minimize the energy of the vortex state, which is a fascinating phenomenon in light of the fact that vortices in type-II samples repel each other and there is no reason for them to conglomerate in clusters (contrary to type-I samples ${ }^{29}$ ). Typically, as a result of repulsion, vortices form Abrikosov lattice or ringlike multivortex states when confined in a mesoscopic sample. In the presence of a weak link, there exists additional quasi-one-dimensional (1D) confinement in the sample, as vortices preferably sit inside the weak link. As a result, not all vortices interact uniformly. The first vortex in the chain interacts weakly with the last one, but very strongly with the nearest one. Therefore, owing to such nonuniform interactions and quasi-1D confinement, the clustering is possible. In addition, the vortex dimer formation is caused by competing interactions, in part owing to the finite size of the sample. If the sample is made larger, dimers will relax closer to equidistant chains.

In the next step, we change the thickness of the weak link while keeping the anisotropy coefficient $\zeta$ fixed at 10 . The results for a weak-link thickness of $30 \mathrm{~nm}$ are shown in Fig. 6(a) and for 70-nm thickness in Fig. 6(b). For a thinner weak link, fewer vortices get inside the link, and correspondingly different vortex configurations are found. For example, in $L=3(\mathrm{a})$, the symmetry in the $z$ direction is broken with only two vortices sitting in the weak link. For the $L=5$ case, we observed a square vortex configuration, with just one vortex inside the weak link. This state actually closely corresponds to the vortex state in the solid cube (Fig. 4), and is only slightly affected by the presence of a weak link. Nevertheless, the novel vortex states owing to the presence of a weak link are still found in this case, exhibiting asymmetry in the $x$ and $z$ directions, as well as the formation of vortex dimers [see the states shown in Fig. 6(a)].

In Fig. 6(b) we have the opposite case, as more vortices reside in the weak link layer owing to its larger thickness. As a consequence, the vortex rearrangement and symmetry breaking occur mainly along the weak link, i.e., in the $x$ direction. Besides vortex dimers, we also found the states with vortex clusters of different size inside the weak link [see the contour plots in Fig. 6(b) for $L=7(\mathrm{a})$ ], where we found not only dimers but vortex trimers as well. As a general trend, owing to the large size of the weak-link layer, it is less energetically costly to break configurational symmetry in the $x$ direction than in the $z$ direction.

In summary of the special vortex features observed in Figs. 5 and 6, we can conclude that, for a given vorticity, the states with more vortices inside the weak link tend to be more stable in high magnetic fields, while those with more vortices inside the superconducting layers tend to be more stable in low magnetic fields. Because the vortices enter (or leave) the sample through the weak link, preexisting vortices inside the weak link can prevent new ones from entering, making the previous state stable in a relatively high magnetic field. In general, the number of vortices inside the weak link depends not only on the size of the weak link and the level of anisotropy, but also on the total number of vortices in the sample, their configuration with respect to the sample 
(a)
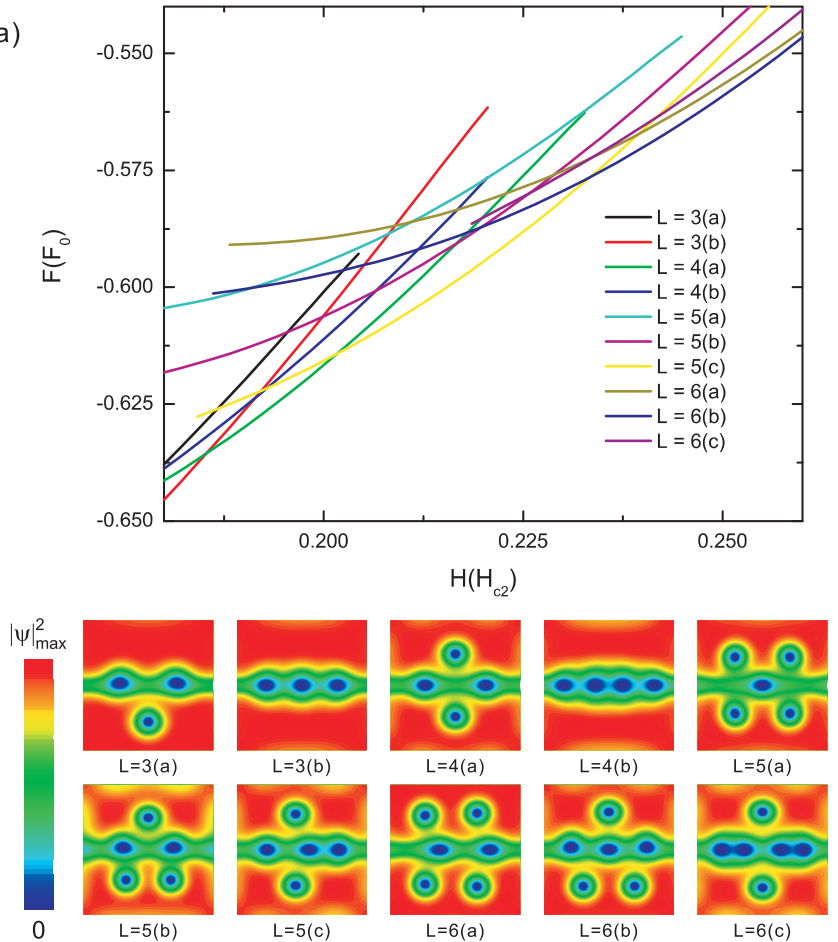

(b)
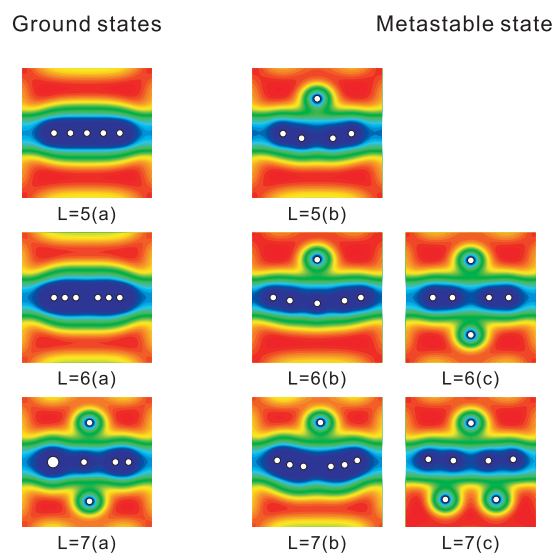

$\mathrm{L}=7(\mathrm{c})$

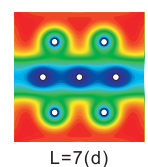

FIG. 6. (Color online) (a) Free-energy curves and corresponding vortex states in a sample with one central weak link of $30 \mathrm{~nm}$ and an anisotropy coefficient $\zeta=10$, in a magnetic field parallel to the $y$ direction. The insets show the contour plots of the Cooper-pair density for selected vortex states. (b) The contour plots of the Cooperpair density for selected vortex states in a sample with a thicker weak link, i.e., for $d=70 \mathrm{~nm}$. The white dots indicate the centers of vortex cores, for clarity (the larger dot denotes a double vortex). The left-hand panel shows the ground-state configurations, and right-hand panel shows the metastable states for a given vorticity.

geometry, and the magnitude of applied magnetic field and the resulting screening currents.

This far, the magnetic field was always parallel to one of the sample sides. In what follows, we investigate the interaction between the vortices and the sample boundary further by tilting the in-plane magnetic field away from the $y$ direction in the $(x, y)$ plane. The results are shown in Fig. 7 for a sample with $d=50 \mathrm{~nm}, \zeta=10$, and the tilt angle of the applied field $\varphi=45^{\circ}$.

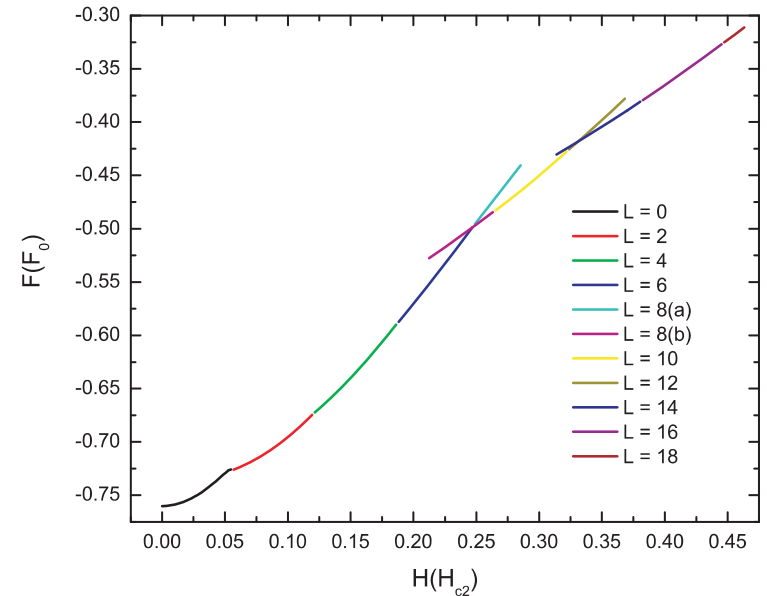

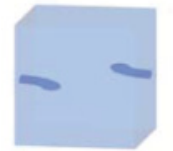

$\mathrm{L}=2$

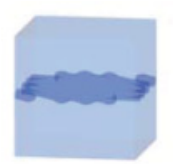

$\mathrm{L}=8(\mathrm{a})$

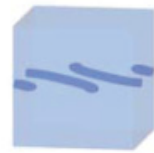

$\mathrm{L}=4$

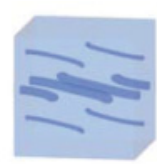

$\mathrm{L}=8(\mathrm{~b})$

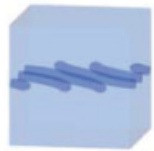

$L=6$

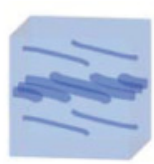

$\mathrm{L}=10$

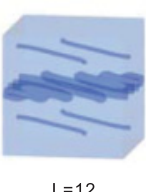

$\mathrm{L}=12$

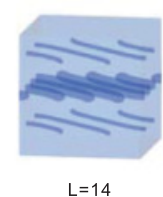

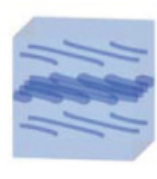

$L=16$

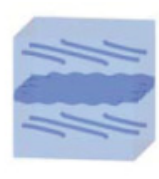

$L=18$
FIG. 7. (Color online) Free energy of the found vortex states by sweeping magnetic field up and down, in a sample with one central weak-link layer of 50-nm thickness and with an anisotropy coefficient $\zeta=10$, in a magnetic field rotated by $\varphi=45^{\circ}$ in the $(x, y)$ plane. The insets show the isoplots of $5 \%$ of the maximal Cooper-pair density for the found vortex states, where isosurfaces outline the shape and arrangement of vortices. The gradual flux entry as a function of magnetic field for $L=2-6$ is shown as an animation in supplementary material (Ref. 33).

With a magnetic field not parallel to the $x$ or $y$ axis, the vortices attempt to change their orientation accordingly. However, they must avoid sharp corners of the sample, where superconductivity is particularly strong (see, e.g., Ref. 25). Namely, in mesoscopic superconductors, the screening effects of Meissner currents that are generated to expel the magnetic field are always maximal in the corners ${ }^{22}$ and vortices, as tubes of magnetic flux avoid those regions. For that reason, the end of each vortex is bent, and the vortex line turns gradually to avoid the corner of the sample. At the boundaries of the sample, the supercurrent can only have the component parallel to the surface (owing to the Neumann boundary condition). Therefore, the vortex endings must be aligned perpendicular to the surface of the sample.

Owing to the perfect symmetry of our sample and the field applied diagonally across it, the $L=1$ state is entirely absent. In increasing magnetic field, once the Meissner state is no longer stable, a vortex should enter the sample and lower the total energy. In this particular case, however, the vortex must 
avoid the sample corner and the resulting vortex state must be asymmetric, or the vortex line must be sufficiently long to avoid two diagonal corners on opposite sides and preserve the symmetry. Either of the two states is energetically expensive; instead, because the sample is geometrically ideal, vortex entry occurs on both sides of the sample simultaneously, in the two corners of the weak-link layer on the diagonal orthogonal to the direction of the applied magnetic field. Finally, two vortices enter the sample, although (by default) having a higher total energy than just a single vortex. Note, however, that the slightest asymmetry in the sample geometry (as is the case in real experimental conditions) would favor the appearance of the single vortex in the ground state.

It is also worth noting that in this group of free-energy curves we discovered several second-order-like transitions between different vortex states. See, for example, the transition from the $L=2$ to $L=4$ state and the subsequent transition from the $L=4$ to $L=6$ state in Fig. 7. This kind of behavior is not expected, because the vortex entry in superconductors is a first-order transition, with an abrupt change in free energy as a function of the applied field. ${ }^{22}$ The latter is owing to the finite barrier for vortex entry, composed of the geometric ${ }^{34,35}$ and the Bean-Livingston (BL) ${ }^{36}$ barrier. In our present system, both those contributions to the barrier for vortex entry are suppressed. The BL barrier is weak inside the weak link, as there superconductivity in general is weak. Nevertheless, if there are vortices already present in the weak link, new vortices will be repelled by the preexisting ones, which will enforce the BL barrier. On the other hand, the geometric barrier is entirely absent in the present geometry of the sample and the direction of the applied field, as vortices enter the sample as pointlike objects at the corners of the weak-link layer. Therefore, the increase of magnetic "pressure" with increasing magnetic field can gradually balance out and overcome the BL barrier (interaction with the existing vortices inside the weak link), and cause the second-order-like manifestation of the vortex entry.

\section{VORTICES IN SAMPLES WITH MULTIPLE WEAK LINKS}

To further expand this study, in this section we increase the number of weak links in the sample to four, and study the behavior of this system in a tilted magnetic field [see Fig. 1(b)]. The thickness of the weak-link layers is taken as $d=30 \mathrm{~nm}$, and the anisotropy coefficient is kept at $\zeta=10$. We apply a magnetic field parallel to the weak-link planes but deviating by $\varphi=45^{\circ}$ from the $y$ axis, as was considered previously in Fig. 7. The obtained free-energy curves as a function of the magnetic field, with insets illuminating different vortex states, are shown in Fig. 8.

In the discussion of Fig. 7, we emphasized that transitions between vortex states can appear as second-order ones. This is not the case for low vorticity in Fig. 8. Namely, because there are four weak links in the sample, each new vortex can enter the sample at a different layer without feeling strong repulsion from the preexisting vortices. However, this does not suggest that the interaction between vortices across weak links can be neglected. In fact, such an interaction can be clearly observed in the analysis of the vortex distribution in

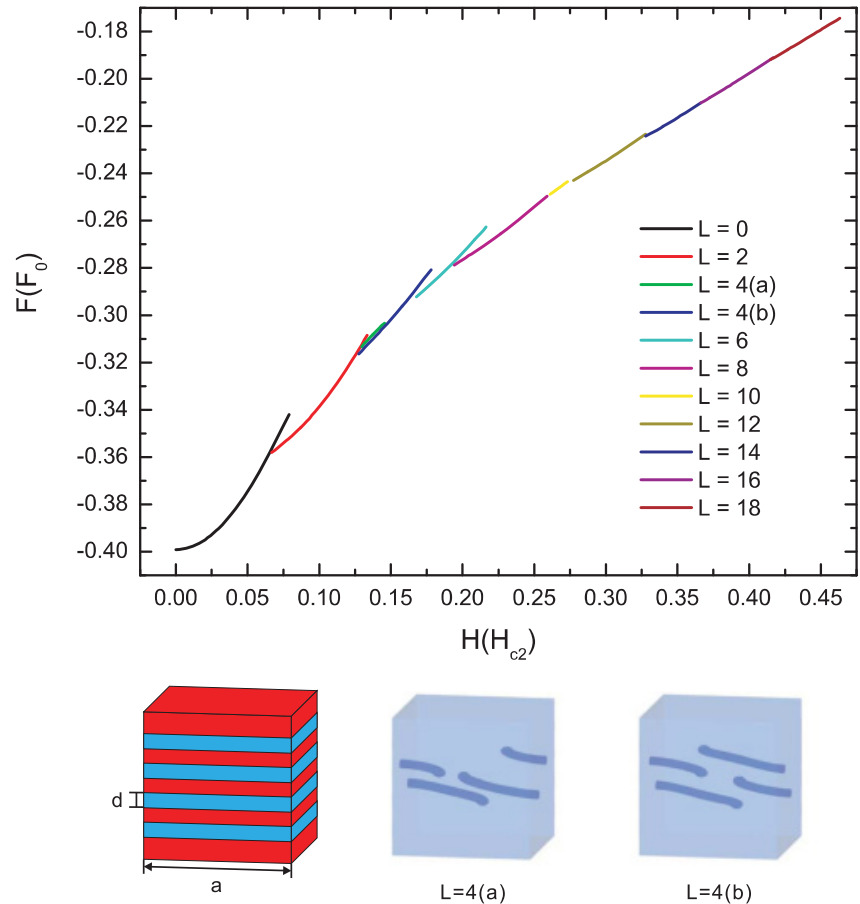

FIG. 8. (Color online) Free energy of selected vortex states in a sample with four weak links of thickness $d=30 \mathrm{~nm}$ and with an anisotropy coefficient $\zeta=10$, in a magnetic field rotated by $\varphi=45^{\circ}$ in the $(x, y)$ plane. The insets show the isoplots of $5 \%$ of the maximal Cooper-pair density for the selected states, where isosurfaces outline the vortices. The shown vortex states are obtained by sweeping the magnetic field up and down in the considered field-range. For the transition between states 4(a) and 4(b) and all found vortex states, please consult supplementary material (Ref. 33).

adjacent weak links. An example is presented in the insets of Fig. 8, showing two different states found for $L=4$. In the $L=2$ state, two vortices enter simultaneously one of the central layers. Subsequently, the two new vortices penetrate the above layer, but cannot take the central position in the layer owing to the repulsion from the two vortices already in the lower layer. This constitutes one $L=4$ state [labeled (a) in Fig. 8], stable in the low magnetic field. However, an increasing magnetic field compresses vortices more toward the center of the sample, owing to the increasing Meissner currents at the sample boundaries. As vortices approach each other, they interact more also with vortices in an adjacent weak link. This repulsion prevents the vortices passing over one another, and leads to a transition to a new $L=4$ state, in which two vortices that are closest to the center of the sample are in two different weak links [state labeled (b) in Fig. 8]. This (and similar) rearrangement is a first-order transition, and therefore observable in experiment. These calculations in a parallel field have a certain connection to the previously intensively studied case of $\mathrm{Bi}-2212$ mesa structures that are always in the mesoscopic limit with respect to the long Josephson penetration depth and the London penetration length in the $c$-axis direction. A similar metastability of the vortex configurations (fluxon modes) was observed there. ${ }^{37,38}$

To further investigate the structure of vortices crossing a set of weak links, we now apply to our sample with four weak 


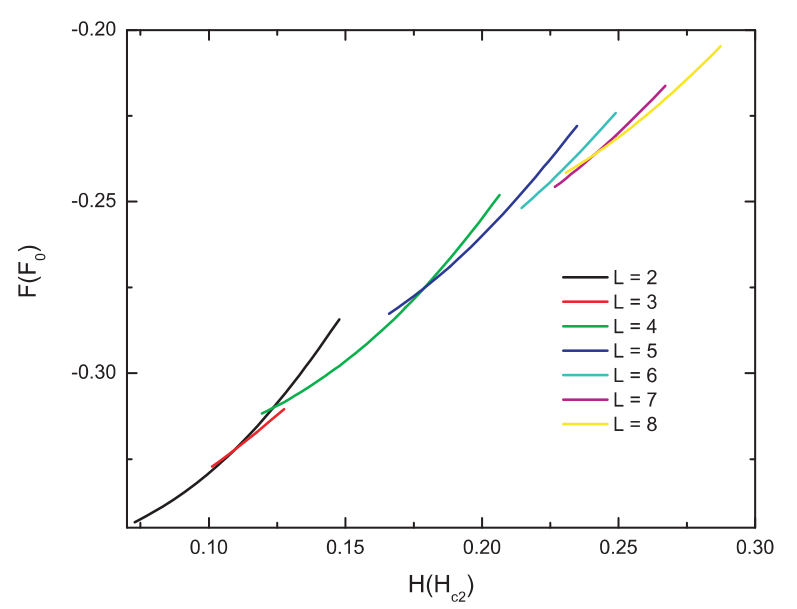

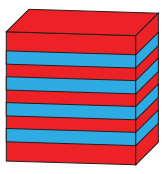

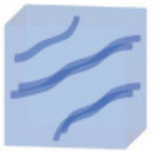

$\mathrm{L}=5$

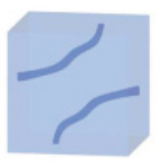

$L=2$

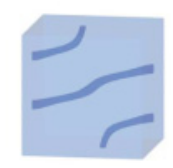

$L=3$

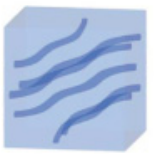

$L=6$

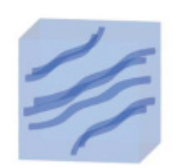

$\mathrm{L}=7$

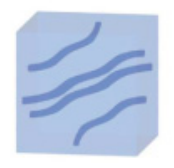

$L=4$

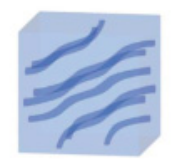

$L=8$
FIG. 9. (Color online) Free energy of selected vortex states in a sample with four weak links of thickness $d=30 \mathrm{~nm}$ and with an anisotropy coefficient $\zeta=10$, in a magnetic field tilted by $\theta=60^{\circ}$ in the $(x, z)$ plane. The insets show the isosurfaces of $5 \%$ of the maximal Cooper-pair density for the selected vortex states. The shown vortex states are obtained by sweeping the magnetic field up and down in the considered field-range. For all found vortex states, please consult supplementary material (Ref. 33).

links a tilted magnetic field in the $(x, z)$ plane. The results are shown in Fig. 9 for the tilt angle $\theta=60^{\circ}$ with respect to the $z$ axis, where selected vortex states and their energies are presented as a function of the applied field. The first conclusion we can draw is that vortices attempt to maximize their length within the weak links. However, for a sufficiently acute $\theta$ angle and a large component of the magnetic field perpendicular to weak links, i.e., along the $z$ axis, vortices have to cross through superconducting regions and vortex kinks are formed, the 3D analogs of pancake vortices in HTSs (see the insets of Figs. 9 and 10).

If we increase the tilt angle $\theta$ of the magnetic field, the vortices will change their orientation accordingly. As a consequence, the vortex strings will become longer in weak links, and vortex fragments in the fully superconducting regions will tilt more with respect to the $z$ axis, and elliptically deform in the $(x, y)$ plane. To illustrate this, we calculated the length of vortex strings in weak links and the ratio of diameters of vortex fragments within fully superconducting regions in the $x$ and $y$ directions for three levels of anisotropy, namely, $\zeta=5$, 10, and 20. Our results (shown in Fig. 10) clearly demonstrate that the length of vortex strings in weak links and the deformation of the vortex fragments in fully superconducting regions both increase with $\theta$. However, neither of them significantly depends on anisotropy. This suggests that the 3D
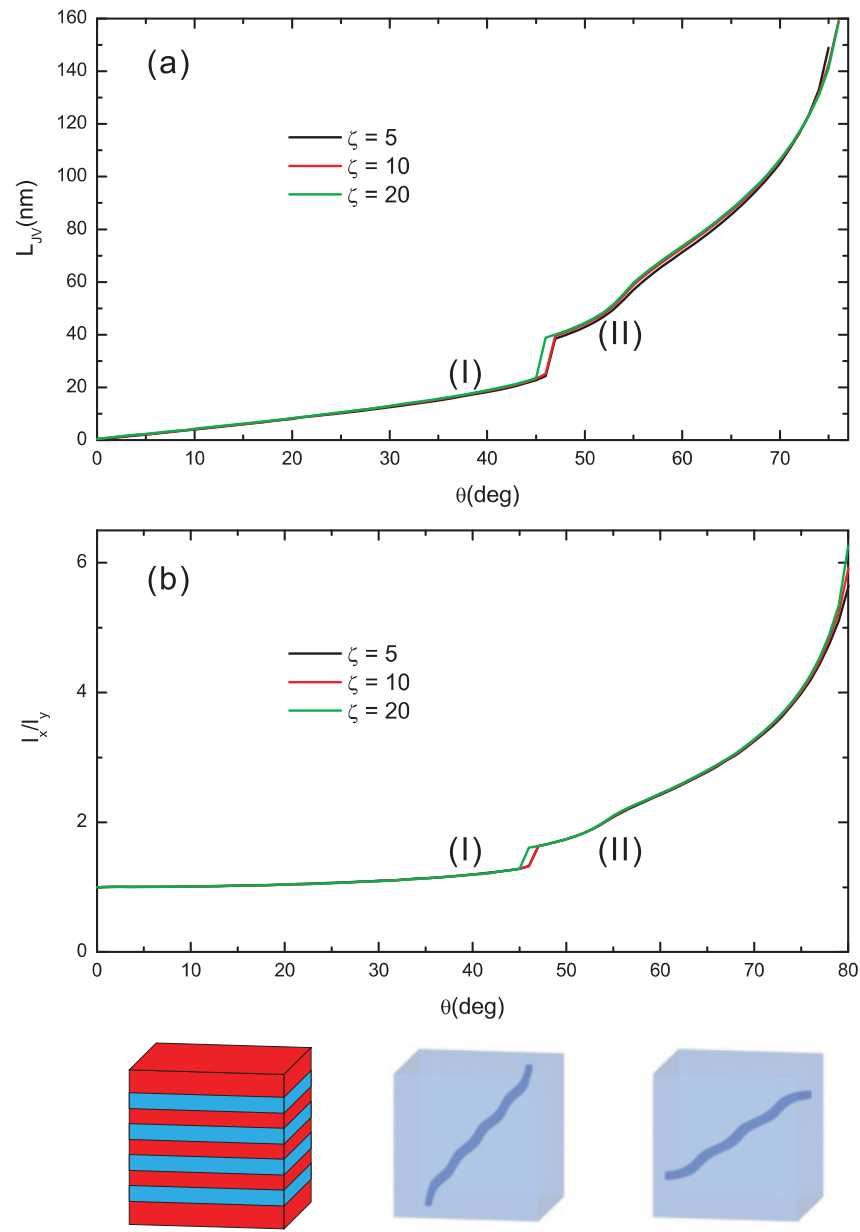

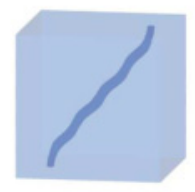

(I)

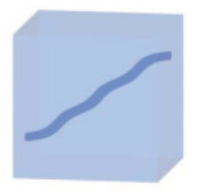

(II)
FIG. 10. (Color online) (a) Dependence of the length of vortex strings in weak links of the $3 \mathrm{D}$ curved vortex line on the tilt angle $\theta$ of the applied field, in a sample with four weak links of 30-nm thickness, for different anisotropy coefficients (the magnitude of the magnetic field is fixed at $0.103 H_{c 2}$ ). (b) The dependence of the deformation of vortex fragments within fully superconducting regions in the $(x, y)$ plane (ratio of the corresponding vortex diameters $l_{x}$ and $l_{y}$ ) on tilt angle $\theta$ for the same sample and magnitude of the magnetic field. The insets depict vortex states in two regimes through isosurfaces of 5\% of the maximal Cooper-pair density. The transition between regimes (I) and (II) as a function of the tilt angle of the applied field is shown in supplementary material (Ref. 33).

vortex-line configuration is determined solely by the direction of the applied field and the mutual interaction of the vortex parts in different layers, incorporated into the full elasticity of the vortex line. In other words, weak anisotropy of our sample results in an almost negligible field-locking angle compared to that of a few degrees in extremely anisotropic, layered (quasi-2D) superconductors such as Bi-2212. ${ }^{39}$

In Fig. 10, we also observed a kink at $\theta=45^{\circ}$. The reason for this kink is illustrated in the insets of Fig. 10. Because the vortex line tilts according to the direction of the magnetic field, its endings will move first on the upper surface of the sample, from the interior of the sample toward the boundary, as angle $\theta$ is increased from zero. However, for $\theta \approx 45^{\circ}$, the vortices will avoid the corner of the sample owing to strong 

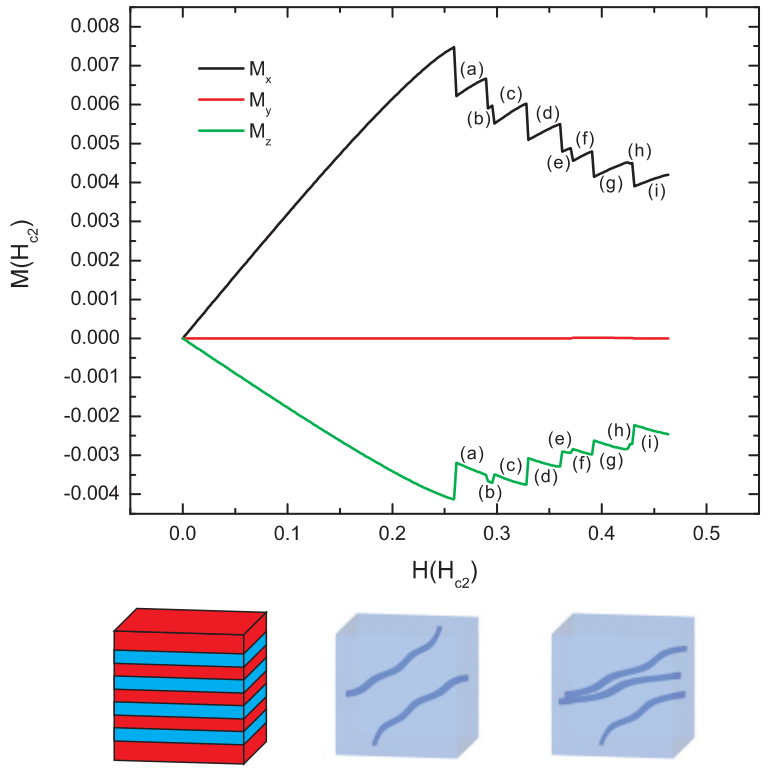

(a) $L=2$

(b) $L=3$

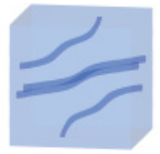

(c) $L=4$

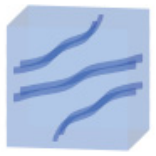

(d) $L=6$

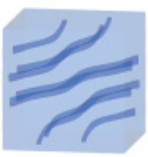

(e) $L=8(a)$

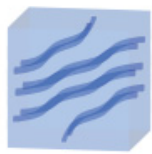

(f) $L=8(b)$

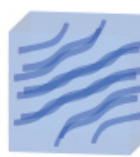

(g) $\mathrm{L}=10(\mathrm{a})$

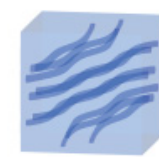

(h) $L=10(b)$

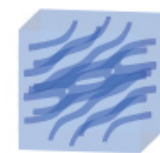

(i) $\mathrm{L}=12$
FIG. 11. (Color online) Magnetization as a function of applied magnetic field, of a sample with four weak links with $d=30 \mathrm{~nm}$ and with an anisotropy coefficient $\zeta=10$, and for a tilt angle of the field $\theta=60^{\circ}$. The insets are the isosurfaces of $5 \%$ of the maximal Cooper-pair density of the selected vortex states, indicated in the magnetization curve. As an example, $\kappa$ is chosen as 0.9 .

Meissner screening there. Ultimately, for $\theta>45^{\circ}$, vortex endings jump over the sample edges to the adjacent surface. After this first-order transition, the length of the vortex strings in weak links and the deformation of vortex fragments in fully superconducting regions will increase faster with increasing tilt angle, as the vortex line falls in plane.

Such a first-order transition leads to a hysteretic behavior of the superconducting quantities as a function of the angle $\theta$, which is certainly observable in experiment. One experimental possibility is to measure the magnetic response of the sample, by, e.g., Hall magnetometry. By solving the two GL equations self-consistently [Eqs. (2) and (3)) with $\kappa=0.9$ ], and by using Eq. (5), we can obtain the magnetization $M(H)$ curves for our sample in a tilted magnetic field, as shown in Fig. 11 for $\theta=60^{\circ}$. We deliberately separate the magnetic response into the Cartesian components, because in the experiment the Hall bar must be placed in a particular 2D plane, and would detect a linear combination of $M_{x}, M_{y}$, and $M_{z}$, depending on its relative position to the sample surfaces.

As is well known, the diamagnetic response of the sample in the Meissner state manifests as the magnetization of the sample increasing with the applied magnetic field. When vortices enter the sample, the magnetization decreases in a steplike manner. In the present case (Fig. 11), the first entering vortex lines penetrate the sample on its $(y, z)$ face, and leave on the $(x, y)$ side (or vice versa). For that reason, the first jump in magnetization, owing to the transition from the Meissner state to the $L=2$ state [see the inset in Fig. 11(a)] is detected in both $M_{z}$ and $M_{x}$ components of the magnetization. However, the further increasing field pushes the vortices toward the $\theta=60^{\circ}$ line, and causes two top vortices in the $L=3$ state to spring entirely to the $(y, z)$ surfaces of the sample [state (b) in Fig. 11], which decreases $\left|M_{x}\right|$ but increases $\left|M_{z}\right|$, as part of the $z$-magnetic response of vortices is lost compared to the previous vortex state (a).

Furthermore, we were also able to magnetically observe the subtle reconfiguration of vortices between different weak links, as is illustrated by states (g) and (h) in Fig. 11. There, two vortices symmetrically penetrating on the $(y, z)$ sides of the sample and leaving on the $(x, y)$ faces shift from the outer to the central weak links, with the up (bottom) vortex part also shifting more centrally in the $(x, y)$ plane. Such transitions leave a decreasing mark in both $\left|M_{x}\right|$ and $\left|M_{z}\right|$, but a fractional one compared to a vortex entry signal (i.e., as a noninteger flux change in units of a flux quantum). Therefore, without going into the fine features of the other vortex states, we hereby conclude that a careful analysis of the corresponding changes in the magnetic response of the sample in different directions can provide information not only about vorticity, but also about the $3 \mathrm{D}$ vortex (re)arrangement, even about the very subtle changes as the ones shown above.

\section{CONCLUSIONS}

To summarize, we investigated the vortex states of a cubic mesoscopic superconductor, layered by one or more weak links-areas with a lower critical temperature, where the change in $T_{c}$ across the sample can be translated into an anisotropy coefficient $\zeta$. As a first finding, we showed the degree of vortex deformation in the weak link when the applied magnetic field is parallel or perpendicular to the weak-link plane, as a function of the level of anisotropy between the superconducting and the weak-link layers. We found that the deformation increases with increasing anisotropy coefficient. Further, we investigated the full vortex phase spectrum and the corresponding free-energy levels in a sample with one central weak link of different thicknesses and anisotropy. We found that the energy barrier for vortices to enter a weak link is smaller than that for vortices to enter a superconducting region. The final distribution of vortices is determined by the competition between the 3D square confinement from the boundary of the sample, the quasi-1D confinement in the weak link, and the interaction between the vortices. As a consequence, we found that asymmetric vortex states are possible. Moreover, vortices inside the weak link can even form dimers and/or trimers as a stable configuration, even though our samples are firmly in the type-II regime and no vortex attraction is present. If the direction of the applied magnetic field is rotated in the plane of the weak-link layer, the vortices attempt to orient accordingly. However, vortices must still adjust their orientation near the boundary of the sample to avoid the corners where the superconductivity is 
strongest, and remain perpendicular to the sample face of entry (exit). Under a particular angle of the rotated field (more specifically, $\varphi=45^{\circ}$ ), vortices enter the sample as pointlike objects at opposite corners of the weak link. In that case, the geometric barrier is fully suppressed, and the BL barrier can be balanced by an applied magnetic field, which causes the vortex entry to be gradual and manifest as a second-order transition, contrary to a conventional first-order picture. This is somewhat changed in the case of multiple weak links, because then the crosstalk across the layers must be taken into account, as vortices interact not only with the vortices present in the same weak link but also with ones in adjacent weak links. This can lead to configurational transformations of the vortex state in multiple weak links, with transitions being of first order.

Under a tilted magnetic field in the $(x, z)$ plane, vortices in our mesoscopic sample are kinked when crossing the fully superconducting regions. We investigated the deformation of the $3 \mathrm{D}$ vortex line owing to the change of direction of the magnetic field and concluded that the deformation of vortex fragments in fully superconducting regions and the elongation of the vortex strings in weak links do not depend on the anisotropy across the sample owing to very small anisotropy and a field-locking angle compared to, e.g., hightemperature superconductors. As the tilt angle of the applied field was changed, we observed a number of interesting vortex transitions, mainly related to the 3D geometry of the sample and the vortex hopping between sides of the sample and also between adjacent weak links. As we showed in our calculated magnetization curves of the sample under a tilted magnetic field, almost all configurational changes in the vortex states leave clear, experimentally detectable signatures in the $M(H)$ loops. A similar conclusion can be expected for the transport measurements, as the dynamic response of the sample will be very sensitive to the shape of the vortex state. Owing to the complexity of the equilibrium vortex phase diagram reported here, we leave the detailed analysis of vortex dynamics in layered mesoscopic superconductors under an applied drive for a separate study.
${ }^{1}$ J. G. Bednorz and K. A. Müller, Z. Phys. 64, 189 (1986).

${ }^{2}$ K. K. Likharev, Rev. Mod. Phys. 51, 101 (1979).

${ }^{3}$ W. E. Lawrence and S. Doniach, in Proceedings of the Twelfth International Conference on Low Temperature Physics, edited by

E. Kanda (Academic Press of Japan, Kyoto, 1971), p. 361.

${ }^{4}$ S. O. Katterwe and V. M. Krasnov, Phys. Rev. B 80, 020502(R) (2009).

${ }^{5}$ P. H. Kes, J. Aarts, V. M. Vinokur, and C. J. van der Beek, Phys. Rev. Lett. 64, 1063 (1990).

${ }^{6}$ L. N. Bulaevskii and J. R. Clem, Phys. Rev. B 44, 10234 (1991).

${ }^{7}$ L. N. Bulaevskii, M. Ledvij, and V. G. Kogan, Phys. Rev. B 46, 366 (1992).

${ }^{8}$ A. E. Koshelev, Phys. Rev. B 48, 1180 (1993).

${ }^{9}$ L. L. Daemen, L. J. Campbell, A. Yu. Simonov, and V. G. Kogan, Phys. Rev. Lett. 70, 2948 (1993).

${ }^{10}$ T. Matsuda, O. Kamimura, H. Kasai, K. Harada, T. Yoshida, T. Akashi, A. Tonomura, Y. Nakayama, J. Shimoyama, K. Kishio, T. Hanaguri, and K. Kitazawa, Science 294, 2134 (2001).

${ }^{11}$ A. Grigorenko, S. Bending, T. Tamegai, S. Ooi, and M. Henini, Nature (London) 414, 728 (2001).

${ }^{12}$ A. E. Koshelev, Phys. Rev. B 68, 094520 (2003).

${ }^{13}$ A. E. Koshelev and I. S. Aranson, Phys. Rev. Lett. 85, 3938 (2000).

${ }^{14}$ M. Machida, Phys. Rev. Lett. 90, 037001 (2003).

${ }^{15}$ M.-H. Bae, H.-J. Lee, and J.-H. Choi, Phys. Rev. Lett. 98, 027002 (2007).

${ }^{16}$ V. V. Moshchalkov, L. Gielen, C. Strunk, R. Jonckheere, X. Qiu, C. V. Haesendonck, and Y. Bruynseraede, Nature (London) 373, 319 (1995).

${ }^{17}$ A. K. Geim, I. V. Grigorieva, S. V. Dubonos, J. G. S. Lok, J. C. Maan, A. E. Filippov, and F. M. Peeters, Nature (London) 390, 259 (1997).

${ }^{18}$ V. A. Schweigert, F. M. Peeters, and P. S. Deo, Phys. Rev. Lett. 81, 2783 (1998)

${ }^{19}$ V. A. Schweigert and F. M. Peeters, Phys. Rev. B 60, 3084 (1999). ${ }^{20}$ V. A. Schweigert and F. M. Peeters, Phys. Rev. B 57, 13817 (1998).

${ }^{21}$ L. F. Chibotaru, A. Ceulemans, V. Bruyndoncx, and V. V. Moshchalkov, Nature (London) 408, 833 (2000).
${ }^{22}$ B. J. Baelus and F. M. Peeters, Phys. Rev. B 65, 104515 (2002).

${ }^{23}$ V. R. Misko, V. M. Fomin, J. T. Devreese, and V. V. Moshchalkov, Phys. Rev. Lett. 90, 147003 (2003).

${ }^{24}$ B. Xu, M. V. Milošević, and F. M. Peeters, Phys. Rev. B 77, 144509 (2008).

${ }^{25}$ V. M. Fomin, J. T. Devreese, and V. V. Moshchalkov, Europhys. Lett. 42, 553 (1998).

${ }^{26}$ M. M. Doria, A. R. de C. Romaguera, and F. M. Peeters, Phys. Rev. B 75, 064505 (2007).

${ }^{27}$ A. R. de C. Romaguera, M. M. Doria, and F. M. Peeters, Phys. Rev. B 75, 184525 (2007).

${ }^{28}$ Y. Chen, M. M. Doria, and F. M. Peeters, Phys. Rev. B 77, 054511 (2008).

${ }^{29}$ G. R. Berdiyorov, A. D. Hernandez, and F. M. Peeters, Phys. Rev. Lett. 103, 267002 (2009).

${ }^{30}$ B. Xu, M. V. Milošević, and F. M. Peeters, Phys. Rev. B 81, 064501 (2010).

${ }^{31}$ B. Rosenstein and D. Li, Rev. Mod. Phys. 82, 109 (2010).

${ }^{32}$ R. Kato, Y. Enomoto, and S. Maekawa, Phys. Rev. B 47, 8016 (1993).

${ }^{33}$ See supplemental material at [http://link.aps.org/supplemental/ 10.1103/PhysRevB.83.104524] for the gradual flux entry for $L=$ 2-6 in Fig. 7, the transition between states 4(a) and 4(b) in Fig. 8, the transition between regimes (I) and (II) in Fig. 9, and all found vortex states in Figs. 8 and 9.

${ }^{34}$ E. Zeldov, A. I. Larkin, V. B. Geshkenbein, M. Konczykowski, D. Majer, B. Khaykovich, V. M. Vinokur, and H. Shtrikman, Phys. Rev. Lett. 73, 1428 (1994).

${ }^{35}$ E. H. Brandt, Phys. Rev. B 59, 3369 (1999).

${ }^{36}$ C. P. Bean and J. D. Livingston, Phys. Rev. Lett. 12, 14 (1964).

${ }^{37}$ V. M. Krasnov, N. Mros, A. Yurgens, and D. Winkler, Physica C 304, 172 (1998).

${ }^{38}$ V. M. Krasnov, V. A. Oboznov, V. V. Ryazanov, N. Mros, A. Yurgens, and D. Winkler, Phys. Rev. B 61, 766 (2000).

${ }^{39}$ D. E. Farrell, S. Bonham, J. Foster, Y. C. Chang, P. Z. Jiang, K. G. Vandervoort, D. J. Lam, and V. G. Kogan, Phys. Rev. Lett. 63, 782 (1989). 